\title{
Prognostic significance of a normal karyotype in adult patients with BCR-ABL1-positive acute lympho- blastic leukemia in the tyrosine kinase inhibitor era
}

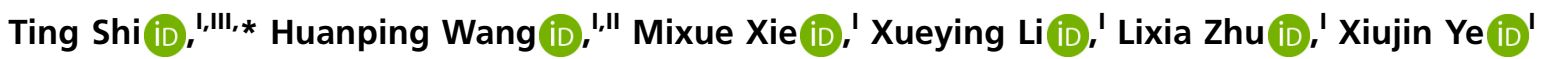 \\ 'Department of Hematology, The First Affiliated Hospital of Medical School of Zhejiang University, Hangzhou, Zhejiang Province, China. "Key Laboratory \\ of Hematology Oncology Diagnosis and Treatment of Zhejiang Province, Hangzhou, Zhejiang Province, China. "'I Program in Clinical Medicine, School of \\ Medicine of Zhejiang University, Hangzhou, Zhejiang Province, China.
}

Shi T, Wang H, Xie M, Li X, Zhu L, Ye X. Prognostic significance of a normal karyotype in adult patients with BCR-ABL1-positive acute lymphoblastic leukemia in the tyrosine kinase inhibitor era. Clinics. 2020;75:e2011

*Corresponding author. E-mail: yxjsunny@zju.edu.cn

OBJECTIVE: The occurrence of cryptic Philadelphia (Ph) chromosome translocation is rare in BCR-ABL1-positive acute lymphoblastic leukemia (BCR-ABL1 $\left.{ }^{+} A L L\right)$ and is of unknown significance in the tyrosine kinase inhibitor (TKI) era.

METHODS: We retrospectively studied a series of adult patients receiving TKI-based therapy to evaluate the prognostic impact of the normal karyotype $(N K)(n=22)$ in BCR-ABL1 ${ }^{+}$ALL by comparison with the isolated Ph ${ }^{+}$ karyotype $(n=54)$.

RESULTS: There were no statistically significant differences in clinical characteristics and complete remission rate between the two groups. Compared with the isolated $\mathrm{Ph}^{+}$group, the NK/BCR-ABL1 ${ }^{+}$group had a higher relapse rate $(55.0 \%$ versus $29.4 \%, p=0.044)$. Overall survival (OS) and disease-free survival (DFS) were significantly shorter in the NK/BCR-ABL1 ${ }^{+}$group than in the isolated $\mathrm{Ph}^{+}$group [median OS: 24.5 versus 48.6 (months), $p=0.013$; median DFS: 11.0 (months) versus undefined, $p=0.008$ ]. The five-year OS and DFS for patients with NK/BCR-ABL1 ${ }^{+}$were $19.2 \%$ and $14.5 \%$, respectively; those for patients with isolated $\mathrm{Ph}^{+}$were $49.5 \%$ and $55.7 \%$, respectively. Thirty-four $(44.7 \%)$ patients underwent allogeneic hematopoietic stem cell transplantation (allo-HSCT) in this study. Among the patients who received allo-HSCT, the median OS and DFS in the NK/BCR$\mathrm{ABL}^{+}$group $(n=9)$ were 35.5 and 27.5 months, respectively, while those in the isolated $\mathrm{Ph}^{+}$group $(n=25)$ were undefined. There was a trend of significant statistical difference in the OS between the two subgroups $(p=0.066)$, but no significant difference in the DFS. Multivariate analysis revealed that NK was independently associated with worse OS and DFS in BCR-ABL1 ${ }^{+}$ALL patients [Hazard ratio (HR) 2.256 (95\% confidence interval (CI), 1.005-5.066), $p=0.049 ; \mathrm{HR} 2.711$ (95\% Cl, 1.319-5.573), $p=0.007]$.

CONCLUSION: Our results suggest that the sub-classification of an NK could be applied in the prognostic assessments of BCR-ABL1 ${ }^{+}$ALL. In addition, allo-HSCT should be actively performed to improve prognosis in these patients.

KEYWORDS: Acute Lymphoblastic Leukemia; BCR-ABL1; Philadelphia Chromosome; Normal Karyotype; Cytogenetics; ABL1 Mutation; Prognosis.

\section{INTRODUCTION}

Acute lymphoblastic leukemia (ALL) is a heterogeneous disease whose genetics are continuously evolving and will likely influence treatment decisions. Philadelphia (Ph) chromosome-positive, or BCR-ABL1-positive ALL, is a separate entity in ALL that accounts for $25-30 \%$ of adult cases and has

Copyright $\odot 2020$ CLINICS - This is an Open Access article distributed under the terms of the Creative Commons License (http://creativecommons.org/licenses/by/ 4.0/) which permits unrestricted use, distribution, and reproduction in any medium or format, provided the original work is properly cited.

No potential conflict of interest was reported.

Received for publication on May 20, 2020. Accepted for publication on July 22,2020

DOI: $10.6061 /$ clinics/2020/e2011 an unfavorable prognosis $(1,2)$. Cytogenetic analysis and molecular screening at the time of diagnosis showed that the results of these patients were quite heterogeneous, including some patients with the $\mathrm{Ph}$ chromosome as the sole abnormality, additional cytogenetic abnormalities, a complex-/ variant-Ph chromosome, or a cryptic Ph chromosome with $\mathrm{BCR}-\mathrm{ABL} 1$ rearrangement $\left(\mathrm{Ph}^{-} / \mathrm{BCR}-\mathrm{ABL} 1^{+}\right)$. An ALL patient with $\mathrm{Ph}^{-} / \mathrm{BCR}-\mathrm{ABL1}{ }^{+}$is quite rare and represents a unique clinical subtype. At present, the mechanism of cryptic Ph chromosome formation in BCR-ABL1 ${ }^{+}$leukemia is mainly derived from chronic myeloid leukemia (CML) research. Two major mechanisms were proposed: (1) there is a direct insertion between chromosome 9 and 22, that is, the protooncogene $A B L 1$ is directly inserted into the BCR region and vice versa, but the former is more common; and (2) there are two sequential translocations including the classic $(9,22)$, followed by reverse translocation with each other, another 
chromosome, or both, thereby restoring the normal chromosome morphology $(3,4)$.

At the time of disease diagnosis, cytogenetic analysis showed that the cryptic $\mathrm{Ph}$ chromosome translocation could present with a normal karyotype (NK) in approximately $3.0 \%-7.7 \%$ of CML patients, as well as a relatively small proportion of BCR-ABL1 ${ }^{+}$ALL patients (5-10). The prognosis of NK in CML patients has been reported, and the results in the tyrosine kinase inhibitor (TKI) era were controversial $(4,11-13)$. However, its role has not been systematically elucidated in adult patients with BCR-ABL1-positive ALL.

Therefore, we retrospectively analyzed the cytogenetic abnormalities in adult patients with BCR-ABL1 ${ }^{+}$ALL receiving chemotherapy plus TKIs to systematically investigate the different clinical implications for cases with NK/ BCR-ABL1 ${ }^{+}$and isolated $\mathrm{Ph}^{+}$.

\section{MATERIALS AND METHODS}

\section{Patients}

We reviewed all patients who were newly diagnosed with BCR-ABL1 ${ }^{+}$ALL and had available data on conventional cytogenetics at diagnosis at our institution between 01/2010 and $12 / 2018$. Only patients with an adequate number of analyzed metaphases $(\geqslant 20)$ during conventional cytogenetic detection and receiving TKI-based therapy in the NK/BCR$\mathrm{ABL1}^{+}(\mathrm{n}=22)$ and isolated $\mathrm{Ph}^{+}(\mathrm{n}=54)$ groups were included in the study $(n=76)$. All the patients met the diagnostic criteria for ALL according to the World Health Organization classification. The patients underwent morphological examination, immunophenotype analysis by flow cytometry, cytogenetic analysis by routine R-banding with karyotype analysis, and leukemia fusion gene screening by multiplex nested reverse transcriptase-polymerase chain reaction (RT-PCR). Fluorescence in situ hybridization (FISH) was examined using BCR/ ABL dual-color dual-fusion translocation Probes (Abbott, USA). Probe and slide preparation, as well as hybridization and washing steps, were performed according to the manufacturer's protocols. Quantitative RT-PCR was used to determine the BCR-ABL1/ABL1 ratio, as well as the BCR-ABL1 positivity status during the whole process of treatment. Cytogenetic analysis for relapse or TKI resistance was performed at the discretion of the treating physician. The ABL1 kinase domain mutation analysis was conducted at the timing of relapsed or refractory status by direct Sanger sequencing. All patients were 16 years or older, and none of them had a history of malignant disease involving CML and myeloproliferative diseases. The study was approved by the Institutional Review Board of the First Affiliated Hospital of Zhejiang University. All procedures performed in studies involving human participants were in accordance with the ethical standards of the institution, national research committee, and 1964 Helsinki declaration and its later amendments or comparable ethical standards.

\section{Treatment and response definitions}

All patients were treated with the standard $\mathrm{VDCP} \pm \mathrm{L}$, $\mathrm{VDP} \pm \mathrm{L}$, or Hyper-CVAD regimes plus TKIs, and chemotherapy comprising vincristine $(\mathrm{V})$, daunorubicin or doxorubicin (D/A), L-asparaginase or asparaginase (L), cyclophosphamide (C), and steroid treatment (prednisone, or dexamethasone) $(\mathrm{P} / \mathrm{D})$. After complete remission (CR), intensive consolidation therapy was based on high-dose methotrexate, cytarabine, cyclophosphamide, and steroid treatment. The incorporation of
TKIs into the therapeutic regime was continuous as maintenance therapy. Triple intrathecal therapy (methotrexate, cytarabine, and dexamethasone) was used for central nervous system prophylaxis. Allogeneic hematopoietic stem cell transplantation (allo-HSCT) was performed according to donor availability and the economic condition of the recipient. Response and relapse assessment were defined according to the ALL criteria of the National Comprehensive Cancer Network (NCCN) (14). Disease-free survival (DFS) was measured at the time of CR until relapse, death, or last follow-up (December 31, 2019). Overall survival (OS) was calculated from the time of disease diagnosis until death or last follow-up. Patients who were losing contact were censored at the last contact date. A total of 12 patients could not be contacted during the follow-up period. The median follow-up duration for the survivors was 21.0 months (range, 1.0-110.0 months).

\section{Statistical analyses}

SPSS 23.0 software was used for the statistical analysis of these data. Descriptive statistics were used to illustrate the clinical features of these cases. Measurement data that conform to a normal distribution are expressed as the means \pm standard deviations, while non-normally distributed continuous data are expressed as the median (range). Categorical variables were compared by the chi-square test, and continuous variables were compared using the t-test or nonparametric analysis (Mann-Whitney U test). The KaplanMeier approach was used to estimate DFS and OS, and the log-rank test was used to compare survival estimates. A Cox regression model was used to identify prognostic variables in patients. Only variables with $p<0.10$ in the univariate analyses were included in the multivariate model; backward elimination was used until all variables showed a $p$-value of $<0.05$. All tests were double-tailed, and $p<0.05$ was considered statistically significant.

\section{RESULTS}

This study included $40(52.6 \%)$ men and $36(47.4 \%)$ women, with an average age of 43.8 years (range, 16-76). The baseline characteristics of the two groups of patients are shown in Table 1. Cytogenetic heterogeneity was not correlated to patient gender, age, white blood cell count, hemoglobin, platelet count, ferritin level, lactate dehydrogenase level, or the type and ratio of BCR-ABL1/ABL expression. In terms of TKI options, three patients in the NK/BCR-ABL1 ${ }^{+}$ and seven patients in the isolated $\mathrm{Ph}^{+}$group were treated with second-generation TKIs (dasatinib) as the first-line treatment. In this study, 34 patients underwent allo-HSCT, and all achieved a post-remission state. Among patients receiving allo-HSCT, there were 9 patients $(40.9 \%)$ in the NK/BCR$\mathrm{ABL}^{+}$group and 25 patients $(46.3 \%)$ in the isolated $\mathrm{Ph}^{+}$ group. No statistically significant difference was observed in the treatment protocols and chemotherapy regimens between the two groups ( $p=0.668$ and undefined, respectively).

In the NK/BCR-ABL + group, the bone marrow samples of two cases were used up and that of one case was of poor quality, resulting in the failure to obtain FISH results for these three patients. Of the remaining 19 patients, the typical abnormal signal pattern, involving chromosomes 22q11 and $9 \mathrm{q} 34$, were detected in 11 patients, and the complex pattern, which involves another chromosome in addition to $22 \mathrm{q} 11$ and $9 q 34$, was detected in 8 patients (Fig. 1). There were only 
Table 1 - Patient characteristics.

\begin{tabular}{|c|c|c|c|c|}
\hline Characteristics & All patients $(n=76)$ & NK/BCR-ABL $+(n=22)$ & Isolated $\mathrm{Ph}^{+}(\mathrm{n}=54)$ & $p$-value \\
\hline Gender (n, M/F) & $40 / 36$ & $12 / 10$ & $28 / 26$ & 0.831 \\
\hline Age $(y s, \bar{x} \pm s)$ & $43.80 \pm 14.98$ & $45.86 \pm 15.32$ & $42.96 \pm 14.91$ & 0.448 \\
\hline WBC count (*10E9/L, range) & $14.10(1.30-403.60)$ & $13.15(2.20-403.60)$ & $16.20(1.30-247.70)$ & 0.208 \\
\hline $\mathrm{Hb}(\mathrm{g} / \mathrm{L}, \overline{\mathrm{x}} \pm \mathrm{s})$ & $102.74 \pm 26.42$ & $105.11 \pm 19.58$ & $101.78 \pm 28.86$ & 0.622 \\
\hline PLT count (*10E9/L, range) & $41.00(2.00-405.00)$ & $25.00(8.00-309.00)$ & $48.00(2.00-405.00)$ & 0.214 \\
\hline LDH (U/L, range) & $513.00(151.00-7209.00)$ & $508.00(164.00-4200.00)$ & $513.00(151.0-7209.00)$ & 0.516 \\
\hline Ferritin (ng/ml, range) & 794.45 (93.00-20985.50) & $580.90(93.00-3718.50)$ & $862.80(170.40-20985.50)$ & 0.218 \\
\hline Blast in BM (\%, range) & $82.00(22.00-96.00)$ & $83.00(37.50-96.00)$ & $78.50(22.00-96.00)$ & 0.312 \\
\hline BCR-ABL/ABL $(\%, \bar{x} \pm s)$ & $61.52 \pm 24.90$ & $61.47 \pm 26.11$ & $61.54 \pm 24.65$ & 0.992 \\
\hline BCR-ABL isoform (n, p190/p210) & $43 / 33$ & $15 / 7$ & $28 / 26$ & 0.193 \\
\hline CNS involvement $(n)$ & 5 & 1 & 4 & 1.000 \\
\hline T315I mutation $(n)$ & 12 & 6 & 6 & 0.094 \\
\hline \multicolumn{5}{|l|}{ Treatment protocol (n) } \\
\hline Chem + TKIs & 42 & 13 & 29 & 0.668 \\
\hline Chem + TKIs + allo-HSCT & 34 & 9 & 25 & \\
\hline \multicolumn{5}{|l|}{ Chemotherapy regimen $(n)$} \\
\hline $\mathrm{VDP} \pm \mathrm{L}$ & 8 & 0 & 8 & - \\
\hline $\mathrm{VDCP} \pm \mathrm{L}$ & 61 & 19 & 42 & \\
\hline Hyper-CVAD & 7 & 3 & 4 & \\
\hline
\end{tabular}

Note: M-male; F-female; WBC—white blood cell; Hb—hemoglobin; PLT_Platelet; LDH—lactate dehydrogenase; BM-bone marrow; C— chemotherapy; CNS-central nervous system; TKIs-tyrosine kinase inhibitors; allo-HSCT-allogeneic hematopoietic stem cell transplantation.
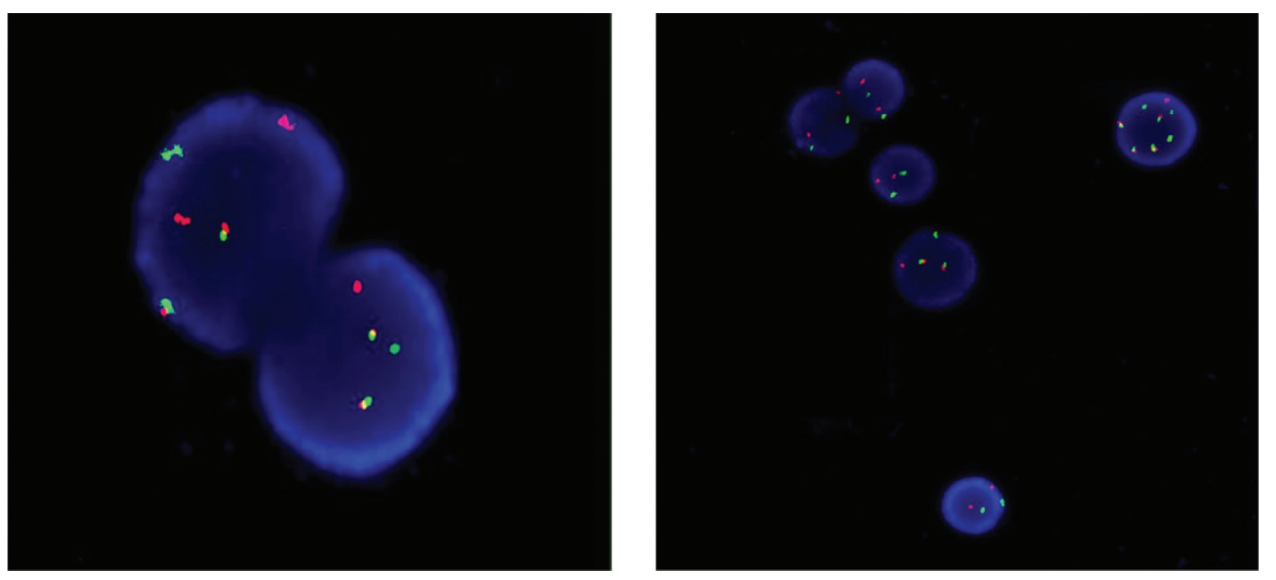

Figure 1 - Fluorescence in situ hybridization (FISH) shows yellow fusion signal (BCR-ABL1 fusion gene) and separate red (ABL1), green $(B C R)$ signals in interphase nuclei in two patients with normal karyotype (NK).

four patients in the $\mathrm{NK} / \mathrm{BCR}-\mathrm{ABL} 1^{+}$group who underwent cytogenetic examination at the time of relapse, and all presented with an NK. ABL1 kinase mutations were detected in 8 and 11 patients in the NK/BCR-ABL1 ${ }^{+}$and the isolated group, respectively. A spectrum of 9 types of amino acid substitutions at 8 different residues were detected, including T315I ( $n=12)$, E255K/V ( $n=3 / 3)$, G250E $(n=1)$, Y253H (n=1), Q252H $(n=1)$, E450A $(n=1)$, F317L $(n=1)$, and F486S $(n=1)$. It is remarkable that T315I was, by far, the most frequent mutation, accounting for $63.2 \%(12 / 19)$ of all resistant cases. Of 19 patients with the ABL1 mutation, 14 (73.7\%) patients had a single mutation, and $5(26.3 \%)$ patients had two types of mutations. There was no statistical difference in the proportion of T315I mutation between the two groups. In the NK/BCR-ABL1 ${ }^{+}$group, the T315I mutation occurred in two patients after imatinib use, one patient after the first-line selection of dasatinib use, and three patients after the failure of imatinib treatment and subsequent switching to dasatinib use; in the isolated $\mathrm{Ph}^{+}$group, the T315I mutation occurred in one patient after imatinib use, one patient after the first-line use of dasatinib, and four patients after the failure of imatinib treatment and subsequent switching to dasatinib or nilotinib use.

In this study, one patient in each of the two groups was losing c after the first induction chemotherapy, and one patient in the isolated $\mathrm{Ph}+$ group died of intracranial hemorrhage during the first induction chemotherapy. The efficacy evaluation of the three patients was not completed. However, the efficacy in 73 patients, including 21 patients in the NK/BCR-ABL1 ${ }^{+}$group and 52 patients in the isolated $\mathrm{Ph}^{+}$group, was evaluated. After the first induction therapy, 19 patients in the NK/BCR-ABL1 ${ }^{+}$group obtained CR $(19 / 21,90.5 \%)$, and 47 patients in the isolated $\mathrm{Ph}^{+}$group obtained CR $(47 / 52,90.4 \%)$. All patients who selected dasatinib as the first-line treatment obtained CR after the first induction chemotherapy. There was no statistical difference in the CR rate between the two groups $(p=1.000)$. Of 73 patients, 1 patient in each group did not achieve remission after repeated induction chemotherapy, and follow-ups were conducted with 71 patients with regard to their recurrence. A total of 11 patients in the NK/BCR-ABL ${ }^{+}$group had recurrences at a rate of $55.0 \%$, and 15 patients in the isolated 
$\mathrm{Ph}^{+}$group had recurrences at a rate of $29.4 \%$, which was significantly lower than the corresponding values in the NK/ $\mathrm{BCR}-\mathrm{ABL} 1^{+}$group $(p=0.044)$. In addition, there were $7(7 / 11$, $63.6 \%)$ and $8(8 / 15,53.3 \%)$ recurrent patients with the ABL1 mutation in the NK/BCR-ABL1 ${ }^{+}$and isolated $\mathrm{Ph}^{+}$ groups, respectively.

The median OS and DFS of all patients were 32.8 months (range, 0.3-110.0 months) and 27.0 months (range, 1.5-109.0 months), respectively. The OS and DFS in the NK/BCR$\mathrm{ABL1}^{+}$group were significantly shorter than those in the isolated $\mathrm{Ph}^{+}$group (median OS: 24.5 versus 48.6 months, $p=0.022$; median DFS: 11.0 months versus undefined, $p=0.008$ ) (Figure $2 \mathrm{a}$ and $\mathrm{b}$ ). The 5-year OS and DFS in the NK/BCRABL1 $^{+}$group were $14.5 \%$ and $19.2 \%$, respectively, while those in the isolated $\mathrm{Ph}^{+}$subgroup were $49.5 \%$ and $55.7 \%$, respectively.

The OS and DFS were longer in patients who underwent allo-HSCT than in those who did not (median OS: undefined versus 18.5 months, $p<0.0001$; median DFS: undefined versus 13.0 months, $p=0.0002$ ) (figure not shown). Further analysis of the survival difference between the two groups with or without allo-HSCT showed that the median OS and DFS of allo-HSCT patients in the NK/BCR-ABL1 ${ }^{+}$group were 35.5 and 27.5 months, respectively, and the median OS and DFS of patients who did not receive allo-HSCT were 11.3 and 9.5 months, respectively. The comparisons were statistically different ( $p=0.003$ and $p=0.007$, respectively) (Figure $3 \mathrm{a}$ and $\mathrm{b}$ ).

Allo-HSCT patients in the isolated $\mathrm{Ph}^{+}$group also had significantly better OS and DFS than patients who did not receive allo-HSCT (median OS: undefined versus 23.0 months, $p=0.0003$; median DFS: undefined versus 17.3 months, $p=$ 0.008 ) (Figure $3 \mathrm{c}$ and $\mathrm{d}$ ).

Of patients who underwent allo-HSCT, there was no significant difference in OS and DFS between the NK/BCR$\mathrm{ABL}^{+}$group and the isolated $\mathrm{Ph}^{+}$group (median OS: 35.5 months versus undefined; $p=0.066$; median DFS: 27.5 months versus undefined, $p=0.209$ ) (Figure $4 \mathrm{a}$ and $b$ ).

Factors influencing OS and DFS in adult patients with $\mathrm{BCR}_{-\mathrm{ABL} 1}{ }^{+}$ALL were analyzed by the univariate and multivariate cox regression analysis, as reported in Tables 2 and 3. As shown in Table 2, we found that the NK was the only independent risk factor for OS in adult patients with BCR-ABL1 ${ }^{+}$ALL (Hazard ratio (HR): 2.256; 95\% confidence interval (CI): 1.005-5.066; $p=0.049)$, while undergoing alloHSCT was an independent protective factor for OS (HR: 0.196; 95\% CI: $0.084-0.458 ; p<0.001)$. The results in Table 3

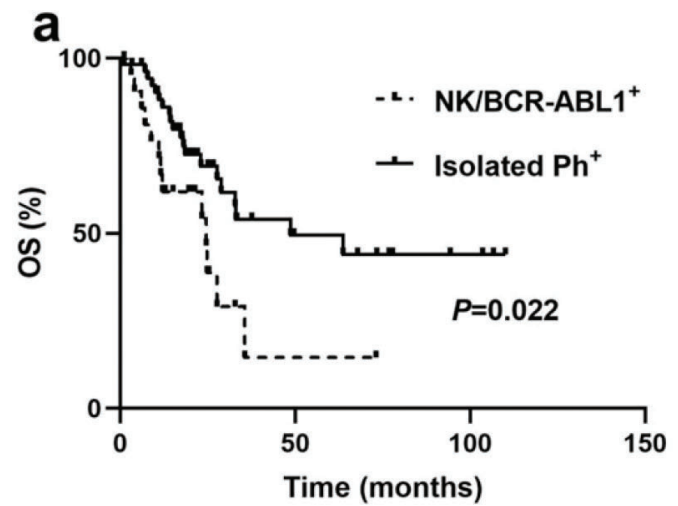

indicate that the NK was the only factor associated with worse DFS (HR: 2.711; 95\% CI: 1.319-5.573; $p=0.007$ ), while undergoing allo-HSCT was the only factor associated with better DFS (HR: 0.224; 95\% CI: 0.099-0.508; $p<0.001$ ).

\section{DISCUSSION}

It is generally accepted that cytogenetic molecular abnormalities are well-recognized and powerful independent prognostic factors for numerous hematologic diseases. The Ph chromosome, creating a novel hybrid gene called BCR-ABL1, was associated with dismal outcomes in adult ALL patients in the pre-TKI era (2). In a few patients, the Ph chromosome translocation was cryptic, owing to some molecular-level insertions or translocations, and thus, could not be detected by G-banding chromosome analysis but could be identified by interphase-FISH and RT-PCR. This suggests that there may be genetic differences between the cryptic and the classic Ph chromosome, and its special formation mechanism may lead to heterogeneity in clinical characteristics and survival prognosis. At the time of disease diagnosis, the cytogenetic analysis showed that a proportion of $\mathrm{Ph}^{-} / \mathrm{BCR}-\mathrm{ABL} 1^{+}$could be found in cases with an NK. Whether the clinical characteristics and prognosis of patients with $\mathrm{Ph}^{-} / \mathrm{BCR}^{-\mathrm{ABL}}{ }^{+}{ }^{+}$ALL or NK/BCR-ABL1 ${ }^{+}$ALL differ from those of $\mathrm{Ph}^{+}$ALL is still unclear, and related reports are lacking.

In this study, we found that the NK/BCR-ABL1 ${ }^{+}$group was similar to the isolated $\mathrm{Ph}^{+}$group, which corroborated the findings obtained in case of CML $(12,15-17)$. With regard to the efficacy of TKIs and prognosis, there are only relevant studies for CML patients. Luatti et al. reported six cases of CML who presented with an NK during cytogenetic analysis at the time of diagnosis, and all the cases received imatinib therapy. Among them, four patients with low-risk Sokal scores achieved complete cytogenetic response at a median time of 24.5 months after receiving imatinib; one patient with a high-risk Sokal score developed imatinib resistance and demonstrated a major molecular response after switching to nilotinib; and only one patient with a moderate-risk Sokal score received allo-HSCT without remission and relapse (11). They concluded that the clinical benefit of TKIs in patients with $\mathrm{NK} / \mathrm{BCR}-\mathrm{ABL} 1^{+}$is similar to that in patients with $\mathrm{Ph}^{+}$, and this view is also recognized by Bennour et al. (13). A study published by Hochhaus et al. also found that like $\mathrm{Ph}^{+}$patients, CML patients with an $\mathrm{NK}$ benefit from

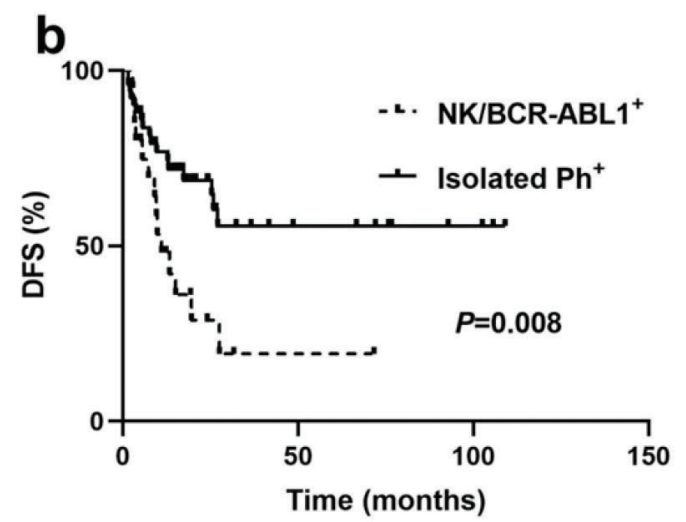

Figure 2 - Outcomes for BCR-ABL1+ patients based on cytogenetic heterogeneity. (a) Overall survival (OS) for patients with NK/BCR$A B L 1^{+}$and isolated $\mathrm{Ph}^{+}$. (b) Disease-free survival (DFS) for patients with NK/BCR-ABL1 ${ }^{+}$and isolated $\mathrm{Ph}^{+}$. 

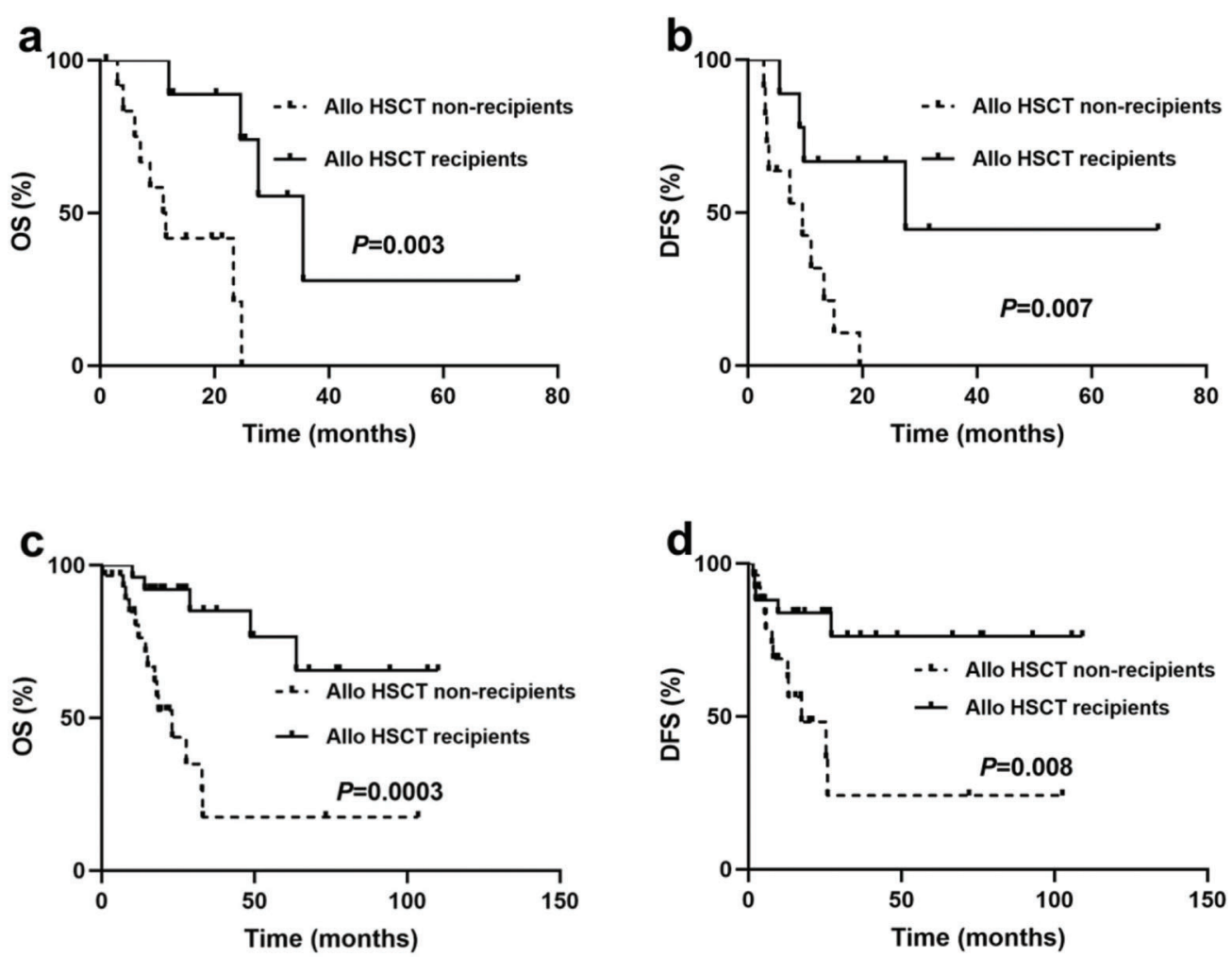

Figure 3 - Outcomes for $B C R-A B L 1^{+}$patients based on cytogenetic heterogeneity and stratified by treatment protocols. (a) Overall survival (OS) and (b) disease-free survival (DFS) for patients with NK/BCR-ABL1 ${ }^{+}$stratified by treatment protocols. (c) OS and DFS (d) for patients with isolated $\mathrm{Ph}^{+}$stratified by treatment protocols.
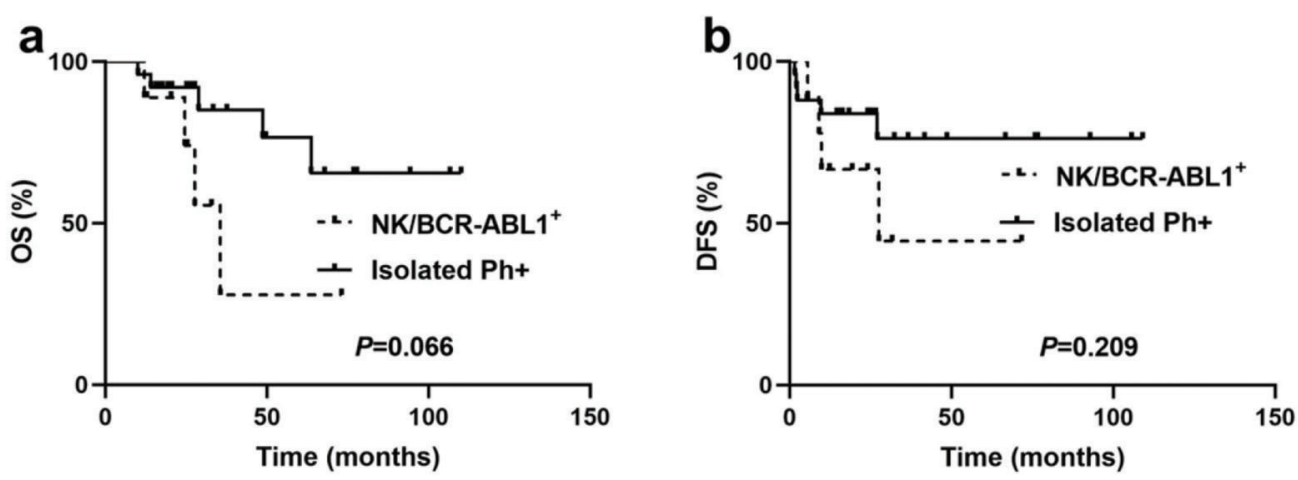

Figure 4 - Outcomes for BCR-ABL1 ${ }^{+}$patients with receiving allo-HSCT based on cytogenetic heterogeneity. (a) Overall survival (OS) for patients with NK/BCR-ABL1 ${ }^{+}$and isolated Ph+. (b) Disease-free survival (DFS) for patients with NK/BCR-ABL1 ${ }^{+}$and isolated Ph ${ }^{+}$.

Table 2 - Risk Factors for Overall Survival in Univariate and Multivariate Analysis.

\begin{tabular}{|c|c|c|c|c|c|c|}
\hline \multirow[b]{2}{*}{ Variable } & \multicolumn{3}{|c|}{ Univariate Analysis } & \multicolumn{3}{|c|}{ Multivariate Analysis } \\
\hline & HR & $95 \% \mathrm{Cl}$ & $p$-value & HR & $95 \% \mathrm{Cl}$ & $p$-value \\
\hline Age $[\geqslant 35 /<35$ (ys)] & 1.856 & $0.834-4.131$ & 0.130 & - & - & - \\
\hline WBC count $[\geqslant 30 /<30(* 10 \mathrm{E} 9 / \mathrm{L})]$ & 1.510 & $0.739-3.081$ & 0.258 & - & - & - \\
\hline PLT count $[\geqslant 30 /<30(* 10 \mathrm{E} 9 / \mathrm{L})]$ & 0.851 & $0.427-1.696$ & 0.647 & - & - & - \\
\hline Cytogenetics (NK/lsolated $\mathrm{Ph}+$ ) & 2.234 & $1.100-4.539$ & 0.026 & 2.256 & $1.005-5.066$ & 0.049 \\
\hline Disease relapse (yes/no) & 3.036 & $1.476-6.245$ & 0.003 & 1.895 & $0.815-4.406$ & 0.138 \\
\hline CNS involvement (yes/no) & 2.655 & $0.905-7.795$ & 0.076 & 2.971 & $0.956-9.235$ & 0.060 \\
\hline allo-HSCT (yes/no) & 0.187 & $0.084-0.415$ & $<0.001$ & 0.196 & $0.084-0.458$ & $<0.001$ \\
\hline
\end{tabular}

Note: WBC—-white blood cell; PLT—platelet; CNS—central nervous system; allo-HSCT—allogeneic hematopoietic stem cell transplantation; Cl—confidence interval; HR-Hazard ratio. 
Table 3 - Risk Factors for Disease-Free Survival in Univariate and Multivariate Analysis.

\begin{tabular}{|c|c|c|c|c|c|c|}
\hline \multirow[b]{2}{*}{ Variable } & \multicolumn{3}{|c|}{ Univariate Analysis } & \multicolumn{3}{|c|}{ Multivariate Analysis } \\
\hline & HR & $95 \% \mathrm{Cl}$ & $p$-value & HR & $95 \% \mathrm{Cl}$ & $p$-value \\
\hline Age $[\geqslant 35 /<35$ (ys)] & 1.844 & $0.788-4.314$ & 0.158 & - & - & - \\
\hline WBC count $[\geqslant 30 /<30(* 10 \mathrm{E} 9 / \mathrm{L})]$ & 1.563 & $0.745-3.278$ & 0.237 & - & - & - \\
\hline PLT count $[\geqslant 30 /<30(* 10 \mathrm{E} 9 / \mathrm{L})]$ & 1.083 & $0.528-2.221$ & 0.827 & - & - & - \\
\hline Cytogenetics (NK/ Isolated Ph +) & 2.524 & $1.239-5.145$ & 0.011 & 2.711 & $1.319-5.573$ & 0.007 \\
\hline CNS involvement (yes/no) & 1.200 & $0.283-5.088$ & 0.805 & - & - & - \\
\hline allo-HSCT (yes/no) & 0.238 & $0.106-0.532$ & $<0.001$ & 0.224 & $0.099-0.508$ & $<0.001$ \\
\hline
\end{tabular}

Note: WBC-white blood cell; PLT_Platelet; CNS—central nervous system; allo-HSCT_allogeneic hematopoietic stem cell transplantation.

nilotinib, as these patients show similar molecular responses (12). On the contrary, three cases of CML with cryptic Ph chromosome reported by Haigh et al. failed to show a major cytogenetic response after imatinib therapy for at least three years, and two of them were shown to have an NK at the initial diagnosis via cytogenetic analysis (4). They believed that compared to classic $\mathrm{Ph}$ chromosomes, cryptic $\mathrm{Ph}$ chromosomes might confer a higher resistance to imatinib.

To our knowledge, this is the first study to examine the impact of an NK on prognosis in adult patients with BCR$\mathrm{ABL}^{+}$ALL using a large mono-centric group of patients in the TKI era. Our analysis showed that despite a similar CR rate in patients with $\mathrm{NK} / \mathrm{BCR}-\mathrm{ABL} 1^{+}$and isolated $\mathrm{Ph}^{+}$, the former has a significantly higher recurrence rate. Our study also found inferior OS and DFS in patients with NK/BCR$\mathrm{ABL}^{+}$compared to those with isolated $\mathrm{Ph}^{+}$. In addition, a trend of a worse prognosis for NK/BCR-ABL1 ${ }^{+}$patients was also observed in those receiving allo-HSCT. We also found that the NK was independently associated with a 2-fold higher risk of relapse or death in patients with BCR-ABL1 ${ }^{+}$ ALL who are treated with chemotherapy plus a TKI. We analyzed the possible reasons for the worse outcome of NK/ $\mathrm{BCR}-\mathrm{ABL} 1^{+}$patients and found that the proportion of ABL1 kinase region mutations in the NK/BCR-ABL1 ${ }^{+}$group was $36.4 \%$, of which T315I mutations accounted for $75 \%$, which was slightly higher than the isolated $\mathrm{Ph}^{+}$group $(20.4 \%$ and $54.5 \%$, respectively). In the first relapse patients, the proportion of patients in the NK/BCR-ABL1 ${ }^{+}$group with the ABL1 mutation was $63.6 \%(7 / 11)$, which is slightly higher than that in case of the isolated $\mathrm{Ph}^{+}$group alone $(53.3 \%$ (8/ $15)$ ). The above results suggest that patients with NK/BCR$\mathrm{ABL1}^{+}$have cytogenetic instability, which may be an important cause of poor prognosis. We postulated that the higher rates of relapse seen in the NK/BCR-ABL1 ${ }^{+}$group might be, in part, due to an increased likelihood of TKI resistance in patients harboring an NK. Unfortunately, we cannot conclude the type and proportion of BCR-ABL1 mutation due to the small number of cases. Thus, future studies should integrate genomic profiling and ABL1 kinase mutation analysis to elucidate the mechanisms mediated by an NK that lead to inferior outcomes in patients with BCR-ABL1 ${ }^{+}$ ALL. At the same time, it is worth exploring further whether there is a difference in the efficacy of each generation of TKIs.

Several limitations of the current study should be mentioned. Firstly, the sample size in the NK/BCR-ABL1 ${ }^{+}$group was relatively small. Secondly, the retrospective nature of this single-center analysis may inevitably affect the strength of the conclusion drawn. Multicenter retrospective analyses or prospective validation in a larger population size are warranted to further determine the role of the NK in BCR$\mathrm{ABL}^{+}{ }^{+} \mathrm{ALL}$ to guide complex management decisions.
Taken together, we demonstrated that the presence of an $\mathrm{NK}$ in patients with BCR-ABL1 ${ }^{+}$ALL receiving chemotherapy plus TKIs was associated with relatively poor outcomes and that an NK could be useful for risk stratification in the near future. Poor-risk NK should be taken into account when developing post-remission strategies in adult patients with BCR-ABL1 ${ }^{+}$ALL.

\section{AUTHOR CONTRIBUTIONS}

Ting shi, Huanping Wang and Mixue Xie contributed equally to this work and share the first authorship. Conception and design: Ting Shi and Mixue Xie. Data collecting: Ting Shi. Statistical analysis: Lixia Zhu and Xueying Li. Manuscript writing: Ting Shi and Mixue Xie. Manuscript Revising: Ting Shi and Huanping Wang. Article reviewing: Xiujin Ye.

\section{REFERENCES}

1. Jabbour E, Pui CH, Kantarjian H. Progress and Innovations in the Management of Adult Acute Lymphoblastic Leukemia. JAMA Oncol. 2018; 4(10):1413-1420. https://doi.org/10.1001/jamaoncol.2018.1915

2. Thomas DA, Faderl S, Cortes J, O'Brien S, Giles FJ, Kornblau SM, et al. Treatment of Philadelphia chromosome-positive acute lymphocytic leukemia with hyper-CVAD and imatinib mesylate. Blood. 2004; 103(12):4396-407. https://doi.org/10.1182/blood-2003-08-2958

3. Al-Achkar W, Liehr T, Wafa A. Insertion of the 3' ABL region into the long arm of chromosome 1 in a Philadelphia chromosome-negative chronic myeloid leukemia case. OncolLett. 2010;1(6):951-954. https://doi.org/ 10.3892/ol.2010.180

4. Haigh S, Cuthbert G. Fluorescence in situ hybridization characterization of different cryptic BCR-ABL rearrangements in chronic myeloid leukemia. Cancer Genet Cytogenet. 2004;155(2):132-7. https://doi.org/ 10.1016/j.cancergencyto.2004.02.026

5. Wrzesie -Ku A, Robak T, Pluta A, Zwoli ska M, Wawrzyniak E, Wierzbowska A, et al. Outcome of treatment in adults with Philadelphia chromosome-positive and/or BCR-ABL--positive acute lymphoblastic leukemia-retrospective analysis of Polish Adult Leukemia Group (PALG). Ann of Hematol. 2006;85(6):366-73. https://doi.org/10.1007/ s00277-006-0099-z

6. Jaso J, Thomas DA, Cunningham K, Jorgensen JL, Kantarjian HM, Medeiros LJ, et al. Prognostic significance of immunophenotypic and karyotypic features of Philadelphia positive B-lymphoblastic leukemia in the era of tyrosine kinase inhibitors. Cancer. 2011;117(17):4009-17. https://doi.org/10.1002/cncr.25978

7. Aldoss I, Stiller T, Cao TM, Palmer JM, Thomas SH, Forman SJ, et al. Impact of Additional Cytogenetic Abnormalities in Adults with Philadelphia Chromosome-Positive Acute Lymphoblastic Leukemia Undergoing Allogeneic Hematopoietic Cell TransplantationBiol Blood Marrow Transplant. 2015;21(7):1326-9. https: / / doi.org/10.1016/j.bbmt. 2015.03.021

8. Akahoshi Y, Mizuta S, Shimizu H, Uchida N, Fukuda T, Kanamori H, et al. Additional cytogenetic abnormalities with Philadelphia chromosome-positive acute lymphoblastic leukemia on allogeneic stem cell transplantation in the tyrosine kinase inhibitor era. Biol Blood Marrow Transplant. 2018;24(10):2009-2016. https://doi.org/10.1016/j. bbmt.2018.06.006

9. Yanada M, Takeuchi J, Sugiura I, Akiyama H, Usui N, Yagasaki F, et al. Karyotype at diagnosis is the major prognostic factor predicting relapsefree survival for patients with Philadelphia chromosome-positive acute lymphoblastic leukemia treated with imatinib-combined chemotherapy. Haematologica. 2008;93(2):287-90. https://doi.org/10.3324/haema tol.11891 
10. Chalandon Y, Thomas X, Hayette S, Cayuela JM, Abbal C, Huguet F, et al. Randomized study of reduced-intensity chemotherapy combined with imatinib in adults with Ph-positive acute lymphoblastic leukemia. Blood. 2015;125(24):3711-9. https://doi.org/10.1182/blood-201502-627935

11. Luatti S, Baldazzi C, Marzocchi G, Ameli G, Bochicchio MT, Soverini S, et al. Cryptic BCR-ABL fusion gene as variant rearrangement in chronic myeloid leukemia: molecular cytogenetic characterization and influence on TKIs therapy. Oncotarget. 2017;8(18):29906-29913. https://doi.org/ 10.18632/oncotarget.15369

12. Hochhaus A, Mahon FX, le Coutre P, Petrov L, Janssen JJWM, Cross NCP, et al. Nilotinib first-line therapy in patients with Philadelphia chromosome-negative/BCR-ABL-positive chronic myeloid leukemia in chronic phase: ENEST1st sub-analysis. J Cancer Res Clin Oncol. 2017;143(7):12251233. https: / / doi.org/10.1007/s00432-017-2359-9

13. Bennour A, Bellâaj H, Ben Youssef Y, Elloumi M, Khelif A, Saad A, et al. Molecular cytogenetic characterization of Philadelphia-negative rearrangements in chronic myeloid leukemia patients. J Cancer Res Clin Oncol. 2011;137(9):1329-36. https://doi.org/10.1007/s00432-011-1002-4
14. Brown PA, Wieduwilt M, Logan A, DeAngelo DJ, Wang ES, Fathi A, et al Guidelines Insights: Acute Lymphoblastic Leukemia, Version 1.2019. J Natl Compr Canc Netw. 2019;17(5):414-423. https://doi.org/10.6004/ jnccn.2019.0024

15. Cortes JE, Talpaz M, Beran M, O'Brien SM, Rios MB, Stass S, et al. Philadelphia chromosome-negative chronic myelogenous leukemia with rearrangement of the breakpoint cluster region. Long-term follow-up results. Cancer. 1995;75(2):464-70. https://doi.org/10.1002/ 1097-0142(19950115)75:2<464::AID-CNCR2820750209>3.0.CO;2-E

16. Martiat P, Michaux JL, Rodhain J. Philadelphia-negative ( $\mathrm{Ph}-$ ) chronic myeloid leukemia (CML): comparison with Ph + CML and chronic myelomonocytic leukemia. The Groupe Français de Cytogénétique Hématologique. Blood. 1991;78(1):205-11. https://doi.org/10.1182/blood. V78.1.205.205

17. Seong D, Kantariian HM, Albitar M, Arlinghaus R, Xu J, Talpaz M, et al. Analysis of Philadelphia chromosome-negative BCR-ABL-positive chronic myelogenous leukemia by hypermetaphase fuorescence in situ hybridization. Ann Oncol. 1999;10(8):955-9. https://doi.org/10.1023/ A: 1008349405763 